\title{
Reconsolidation and Extinction Are Dissociable and Mutually Exclusive Processes: Behavioral and Molecular Evidence
}

\author{
Emiliano Merlo, Amy L. Milton, Zara Y. Goozée, David E. Theobald, and Barry J. Everitt \\ Behavioural and Clinical Neuroscience Institute and Department of Psychology, University of Cambridge, Cambridge CB2 3EB, United Kingdom
}

\begin{abstract}
Memory persistence is critically influenced by retrieval. In rats, a single presentation of a conditioned fear stimulus induces memory reconsolidation and fear memory persistence, while repeated fear cue presentations result in loss of fear through extinction. These two opposite behavioral outcomes are operationally linked by the number of cue presentations at memory retrieval. However, the behavioral properties and mechanistic determinants of the transition have not yet been explored; in particular, whether reconsolidation and extinction processes coexist or are mutually exclusive, depending on the exposure to non-reinforced retrieval events. We characterized both behaviorally and molecularly the transition from reconsolidation to extinction of conditioned fear and showed that an increase in calcineurin (CaN) in the basolateral amygdala (BLA) supports the shift from fear maintenance to fear inhibition. Gradually increasing the extent of retrieval induces a gradual decrease in freezing responses to the conditioned stimulus and a gradual increase in amygdala CaN level. This newly synthesized $\mathrm{CaN}$ is required for the extinction, but not the reconsolidation, of conditioned fear. During the transition from reconsolidation to extinction, we have revealed an insensitive state of the fear memory where NMDA-type glutamate receptor agonist and antagonist drugs are unable either to modulate CaN levels in the BLA or alter the reconsolidation or extinction processes. Together, our data indicate both that reconsolidation and extinction are mutually exclusive processes and also reveal the presence of a transitional, or "limbo," state of the original memory between these two alternative outcomes of fear memory retrieval, when neither process is engaged.
\end{abstract}

Key words: calcineurin; extinction; fear memory; reconsolidation; transition

\section{Introduction}

Persistence or inhibition of aversive and appetitive memories in the brain is determined by retrieval conditions. Following fear conditioning, in which an association forms between a conditioned stimulus (CS) and a footshock [unconditioned stimulus (US)], later presentations of the CS can elicit the conditioned fear response of freezing (Blanchard and Blanchard, 1969). Fully consolidated fear memories, when reactivated by brief CS exposure, return to a labile state that is sensitive to disruption by amnestic agents such as protein synthesis inhibitors. From this labilized state, the fear memory requires reconsolidation to persist in the brain (Nader et al., 2000; Lee et al., 2006). However, if the nonreinforced CS is presented repeatedly, the behavioral outcome is fear memory extinction (Pavlov, 1927), which is characterized by the inhibition of the freezing response to the CS and is also protein synthesis dependent (Vianna et al., 2001). After extinction,

\footnotetext{
Received Sept. 18, 2013; revised Dec. 20, 2013; accepted Dec. 27, 2013.

Author contributions: E.M. designed research; E.M., Z.Y.G., and D.T. performed research; E.M. analyzed data; E.M., A.L.M., and B.J.E. wrote the paper.

This research was supported by a UK Medical Research Council Programme Grant (no. 9536855) to B.J.E., and was conducted in the Department of Psychology and the Medical Research Council/Wellcome Trust Behavioural and Clinical Neuroscience Institute. E.M. was supported by a Royal Society Newton International Fellowship. A.L.M. was partly supported by the Ferreras-Willetts Fellowship in Neuroscience at Downing College, Cambridge University. We thank Dr. Jimena Berni, Dr. Lia Frenkel, and Dr. Gonzalo Urcelay for discussion and helpful comments on the manuscript; and Alan Lyon for technical assistance.

The authors declare no competing financial interests.

Correspondence should be addressed to Dr. Emiliano Merlo, Department of Psychology, University of Cambridge, Cambridge CB2 3EB, UK. E-mail: em454@cam.ac.uk.

DOI:10.1523/JNEUROSCI.4001-13.2014

Copyright $\odot 2014$ the authors $\quad 0270-6474 / 14 / 342422-10 \$ 15.00 / 0$
}

the original fear memory is not erased, but is inhibited by the newly acquired extinction memory (Bouton, 1991). These data illustrate the paradox that persistence of fear through reconsolidation and the loss of fear through extinction are both induced by retrieval of the memory (Eisenberg et al., 2003; Pedreira and Maldonado, 2003). To date, the majority of research has focused on understanding the mechanisms responsible for the two extreme outcomes of retrieval: reconsolidation and extinction (Suzuki et al., 2004; de la Fuente et al., 2011). But it is not known how increasing numbers of CS presentations lead from the termination of reconsolidation (and protection of the original memory) to extinction. The amygdala plays a critical role in the persistence of conditioned fear (Dunn and Everitt, 1988; Campeau and Davis, 1995; LeDoux, 2000). Rapid transitions between states of high and low fear can be induced by a switch in the activation of two distinct subpopulations of neurons in the basolateral amygdala (BLA; Herry et al., 2008), which is therefore a key locus for the transition between persistence and loss of fear.

While the activities of specific kinases exert a positive influence to increase synaptic efficacy (Sweatt, 2004), specific phosphatases exert a negative influence either to reduce [during long-term depression (LTD)] or constrain increases [during long-term potentiation (LTP)] in synaptic efficacy (Mulkey et al., 1994; Ikegami et al., 1996). As proposed for the formation of the original memory (Malleret et al., 2001), the balance between kinases and phosphatases could be pivotal in the establishment of the dominant memory process engaged by different retrieval events. 
The objective of the present study was to investigate the role of the extracellular signal-regulated protein kinase (ERK) and the $\mathrm{Ca}^{2+}$ - and calmodulin-dependent protein phosphatase, calcineurin $(\mathrm{CaN})$ in the BLA on the persistence or inhibition of cued fear memory, and to define the mechanism by which the gradual increase in non-reinforced presentations of the CS shapes the behavioral and molecular transitions between reconsolidation and extinction. We hypothesized that in the amygdala there would be an NMDAR-dependent specific balance between pERK1/2 and CaN levels related to each memory process that underlies the transition from reconsolidation to extinction of fear memory.

\section{Materials and Methods}

Animals. Adult male Lister hooded rats weighing 250-300 g (Charles River) were used. All animals were kept under a $12 \mathrm{~h}$ light/dark cycle (lights off at 7:00 A.M.), and were provided with food and water ad libitum. All animal procedures were conducted in accordance with the UK Animals (Scientific Procedures) Act of 1986.

Surgeries. Rats were anesthetized with ketamine hydrochloride (100 $\mathrm{mg} / \mathrm{kg}$; Ketaset, Fort Dodge Animal Health) and xylazine ( $9 \mathrm{mg} / \mathrm{kg}$; Rompun, Bayer), and implanted with a 22 gauge stainless steel bilateral indwelling guide cannula (Plastics One) aimed at the BLA. The coordinates were $3.6 \mathrm{~mm}$ posterior to bregma, $4.5 \mathrm{~mm}$ lateral to the mid-line, and 3.6 $\mathrm{mm}$ ventral to dura mater. Stainless steel obturators were inserted to maintain patency during recovery and in between infusions.

Intracranial microinfusions. Infusions were performed using a syringe pump connected to injectors (28 gauge, projecting $4 \mathrm{~mm}$ beyond the guide cannula) by polyethylene tubing. Before behavioral testing, animals were habituated to the infusion procedure by the administration of $0.5 \mu \mathrm{l}$ of sterile saline solution per side $(0.25 \mu \mathrm{l} / \mathrm{min})$. Desalted and phosphorothioate end-capped 18-mer sequence oligodeoxynucleotides (ODNs; Sigma-Aldrich) were resuspended in sterile saline solution to a concentration of $10 \mathrm{nmol} / \mu \mathrm{l}$ each sequence. The sequences of the antisense oligos targeting the A subunits of calcineurin were obtained from previous reports (Garver et al., 1999), as follows: calcineurin A1 antisense, 5' -CTC GGA CAT CTC CAG TCA-3'; A2 antisense, 5' -CTC CGG GGC GGC CAT GCT-3' (the italicized codons correspond to the translation initiation complementary site). ODNs with the same composition of bases but in a scrambled (Scr) order were used, as follows: A1 scrambled, 5' -GTC GCA GAT CCT CCA ACT-3'; A2 scrambled, 5' -GCT CGT TAG CCG GCG CGC-3'. Using a basic local alignment search tool (BLAST; Altschul et al., 1997), the antisense ODN sequences showed a significant full alignment only for the respective rat mRNA sequences, while the Scr ODNs failed to fully align with any sequence in the database. A total of $0.5 \mu \mathrm{l}$ of ODN solution per side $(0.25 \mu \mathrm{l} / \mathrm{min})$ was infused $2.5 \mathrm{~h}$ before the first CS of the reactivation or extinction session. The use of short-length ODNs, with only three phosphorothioated bases per end and more than one scrambled control sequence, significantly reduces the possibility of both nontarget (other mRNA sequences) and off-target effects (no Watson-Crick pairs).

Behavioral procedures. Animals were initially individually habituated to the conditioning box (Paul Fray Limited) for $2 \mathrm{~h}$. On the training day, rats were placed in the box and after 25 min received an auditory CS presentation ( $60 \mathrm{~s}$ clicker, $10 \mathrm{~Hz}, 80 \mathrm{~dB}$ ) that was coterminous with the presentation of a scrambled footshock (i.e., a US; $0.5 \mathrm{~mA}, 0.5 \mathrm{~s}$ ) delivered through the grid floor. The training session consisted of two CS-US presentations with an intertrial interval (ITI) of $5 \mathrm{~min}$. Twenty-four hours later, the rats were returned to the box and presented with 1, 4, 7, or $10 \mathrm{CS}$ presentations (ITI $=1 \mathrm{~min}$ ). Depending on the experiment, 24 or $96 \mathrm{~h}$ later animals were again returned to the conditioning box and presented with one CS. All training, CS presentations, and test sessions were video recorded for off-line behavioral analysis. The percentage of time freezing (absence of movement except for breathing) during the 1 min before and during the $1 \mathrm{~min}$ CS was manually scored from the videos by an observer blind to the treatment. Statistical analyses were performed using one-way or repeated-measures ANOVA, and post hoc comparisons were made using Tukey's test.
Drug injection. NMDAR partial agonist D-cycloserine (DCS) and antagonist MK-801 (Sigma-Aldrich) solutions were both freshly prepared with sterile saline solution for intraperitoneal injection $(1 \mathrm{ml} / \mathrm{kg})$. The doses of $15 \mathrm{mg} / \mathrm{kg}$ for DCS and $0.1 \mathrm{mg} / \mathrm{kg}$ for MK-801 were selected on the basis of their facilitatory (DCS) and inhibitory (MK-801) effects on reconsolidation and extinction of fear memory (Lee et al., 2006). The injections of DCS, MK-801, or saline solution were given $30 \mathrm{~min}$ before the CS presentation sessions. Intra-BLA infusions of DCS induce similar effects on memory as the systemic administrations (Walker et al., 2002; Lee et al., 2006; Mao et al., 2006), strongly suggesting that the BLA is one of the main targets of the systemic manipulations of NMDAR used in memory paradigms.

Protein extraction and Western blotting. After CS presentation sessions or $24 \mathrm{~h}$ after training [control (Ctr) groups] rats were killed by carbon dioxide inhalation followed by neck dislocation. The brains were rapidly removed and snap frozen on dry ice before storage at $-80^{\circ} \mathrm{C}$. Samples from the BLA were microdissected using a 0.99 -mm-diameter punching tool from $150-\mu \mathrm{m}$-thick brain sections. To obtain cytoplasmic protein extracts, BLA tissue from each animal was individually disrupted with a Dounce tissue grinder (loose pestle, Wheaton) in $100 \mu$ l of buffer $(10 \mathrm{~mm}$ HEPES, pH 7.9, $1.5 \mathrm{~mm} \mathrm{MgCl}_{2}, 10 \mathrm{~mm} \mathrm{KCl}, 1$ mM DTT, $1 \mu \mathrm{g} / \mathrm{ml}$ Pepstatin A, $10 \mu \mathrm{g} / \mathrm{ml}$ leupeptin, $0.5 \mathrm{~mm}$ PMSF, and $10 \mu \mathrm{g} / \mathrm{ml}$ aprotinin) and centrifuged at $1000 \times g$ for $15 \mathrm{~min}$ at $4^{\circ} \mathrm{C}$. The supernatant (cytoplasmic protein extract) was transferred to a clean tube and stored at $-80^{\circ} \mathrm{C}$. The protein content was determined by Bradford assay. A total of 5-10 $\mu \mathrm{g}$ of cytoplasmic proteins was separated using a 10\% SDS-PAGE and was electrotransferred into a nitrocellulose membrane. Blots were probed with the following: mouse anti-calcineurin (1:2500; catalog \#610260, BD Biosciences); mouse anti-ERK1/2 (1:5000; catalog \#610124, BD Biosciences); rabbit anti-phospho-p44/42 MAPK (ERK1/2; Thr 202/Tyr 204; D13.14.4E; 1:500; Cell Signaling Technology); mouse anti- $\beta$-actin (AC-15; 1:50,000, AbCam); goat anti-rabbit-HRP (1:10,000; Sigma); and rabbit anti-mouse-HRP (1:25,000; Sigma) diluted in Tris-buffered saline solution containing $0.1 \%$ of Tween-20. A chemiluminescent signal was induced using an enhanced chemiluminescent reagent (GE Healthcare), and images were captured using a cooled CCD camera (ChemiDoc-It, UVP). Signal analysis and quantification were performed using ImageJ software (version 1.47a, National Institutes of Health). Each primary antibody working concentration was adjusted to deliver a linear relationship between the amounts of loaded protein in the blot and signal intensity. The optical density (OD) of the bands of interest was measured, and the intensity of the experimental conditions (relative OD) was calculated, normalizing these values to the mean OD of the control group. To control for loading variations, the amount of $\beta$-actin for each sample was used as a normalizing value. Data were analyzed using a one-way ANOVA with Dunnett's test for post hoc comparisons.

\section{Results}

pERK1/2 and calcineurin are differentially associated with the reconsolidation and extinction of fear memory

According to our previous data (Lee et al., 2006), varying the number of non-reinforced CS presentations in animals with fully consolidated auditory fear memory results in the engagement of either reconsolidation or extinction processes. In the first set of experiments, we analyzed the effect of two protocols of unreinforced CS presentation on the freezing response to the fear CS and on the levels of two memory-related proteins, pERK1/2 and CaN.

Two groups of rats were fear conditioned by pairing two presentations of a clicker (CS) with a mild electric footshock (US). As expected, during the training session the percentage of time spent freezing to the CS significantly increased after the first pairing $\left(F_{(1,14)}=122.48, p<0.001, \eta^{2}=0.89 ;\right.$ Fig. $\left.1 A\right)$, with no difference between groups $\left(F_{(1,14)}=1.01, p=0.33\right)$. The following day, each group of animals was presented either with 1 or $10 \mathrm{CS}$ (groups 1CS or 10CS; ITI $=1 \mathrm{~min}$ ). Both groups showed fear memory retention as indicated by a high percentage of time freezing to the auditory CS (pre-CS vs CS1: $F_{(1,14)}=299.91, p<0.001$, 
A

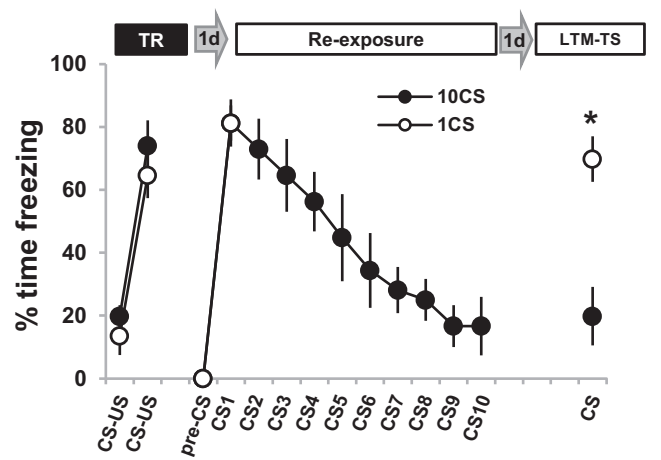

B

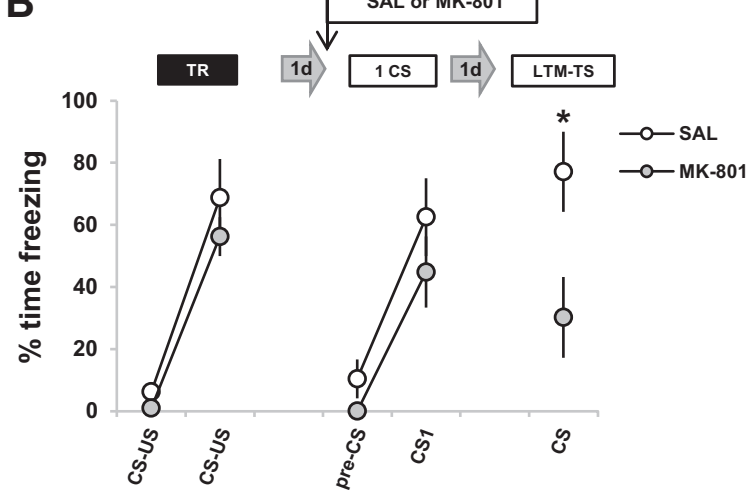

C

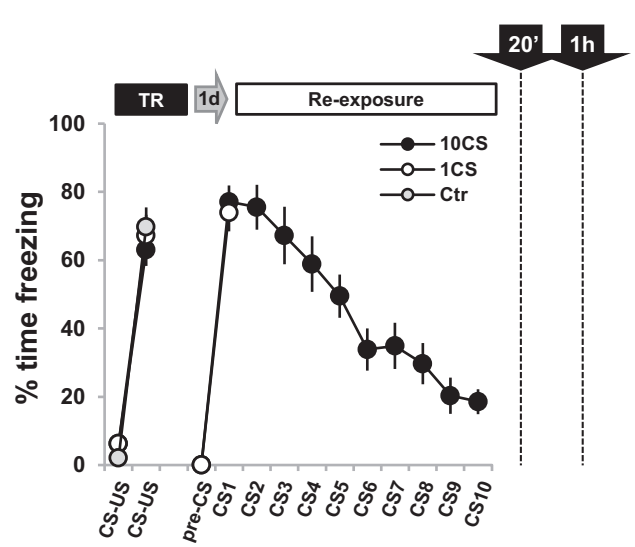

D

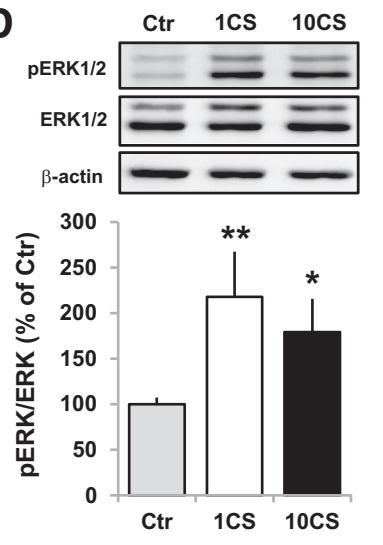

E

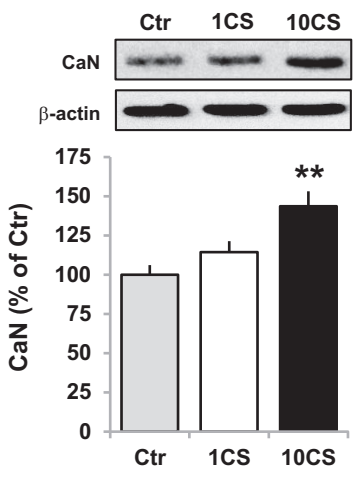

Figure 1. BLA pERK1/2 is increased in reconsolidation and extinction, while CaN levels are only upregulated after extinction. $A$, At training, rats received two CS-US pairings. Twenty-four hours later, the animals were re-exposed to 1 or 10 CSs to reactivate or extinguish the fear memory ( $n=8$ per group). The following day at the retention test, all the animals were presented with one CS. $B$, Twenty-four hours after training, the animals were injected intraperitoneally with either saline solution $(n=4)$ or MK-801 $(0.1 \mathrm{mg} / \mathrm{kg}, n=8) 30$ min before being re-exposed to one CS. During the LTM-TS, fear memory was measured by presentation of one CS. During training, there was an effect of $\mathrm{CS}\left(F_{(1,10)}=73.01, p<0.001, \eta^{2}=0.88\right)$, with no effect of group $\left(F_{(1,10)}=2.57, p=\right.$ 0.14). At reactivation, memory retention was evident since the animals froze significantly more to the fear $C S\left(F_{(1,10)}=25.63, p<0.001, \eta^{2}=0.72\right)$, with no effect of group $\left(F_{(1,10)}=2.19, p=\right.$ 0.17). At LTM-TS, the MK-801 group showed a freezing response that was significantly lower than that in the saline group $\left(F_{(1,10)}=5.13, p<0.05, \eta^{2}=0.34\right)$, indicating the amnesic effect of MK-801 on memory reconsolidation. C, The same behavioral procedure as in $A$, but with the inclusion of a non-reactivated Ctr group that was trained identically to the $1 C S$ and $10 C S$ groups but during the re-exposure session remained in the home cage. Twenty (pERK1/2) or 60 (CaN) minutes after re-exposure, or straight from the home cage (Ctr groups), animals were killed and the cytoplasmic protein extracts from the BLA obtained (all groups, $n=8)$. D, E, Representative Western blot results and analysis showing the cytosolic levels of pERK1/2 (D) or CaN (E) after 1 or $10 C S$ presentations. Data are presented as means \pm SEM. ${ }^{*} p<0.05,{ }^{* *} p<0.01$.

$\left.\eta^{2}=0.95\right)$, with no differences between groups $(F<1)$. In the 10CS group, freezing significantly decreased on presentation of the CSs $\left(F_{(9,63)}=10.52, p<0.001, \eta^{2}=0.60\right)$. Twenty-four hours later, a long-term memory test (LTM-TS) was conducted to measure freezing to the CS in both groups. The 10CS group showed a significant reduction in the percentage of time spent freezing compared with the 1 CS group $\left(F_{(1,14)}=18.04, p<0.001\right.$, $\left.\eta^{2}=0.56\right)$. Given the well established finding that NMDAR antagonism during fear memory reactivation induced by a single CS presentation results in subsequent amnesia (Fig. 1B; Lee et al., 2006), the present findings confirmed that following CS shock fear conditioning, the presentation of one CS results in reconsolidation and thereby maintenance of the fear response to the CS, while 10 presentations of the CS results in extinction and inhibition of the fear response.

We then analyzed the effect of 1 or 10 presentations of the fear CS on the levels of pERK1/2 and CaN in the BLA. Twenty-four hours after fear conditioning, separate groups of rats were exposed to 1 or 10 CSs as before, or remained in the home cage (non-reactivated Ctr group), and were killed after 20 or $60 \mathrm{~min}$ (Fig. 1C). BLA cytoplasmic proteins were prepared and analyzed by Western blots. Other time points after the presentation of 1 or
10 CSs were analyzed, but there was no increase in either pERK1/2 or CaN in BLA cytosolic or nuclear protein extracts (data not shown). The Ctr group used here allowed us to define basal pERK1/2 or CaN levels in BLA $24 \mathrm{~h}$ after training, but before any putative change induced by CS presentations. While pERK1/2 levels were increased after both $1(p<0.01)$ or $10\left(p<0.05\right.$; one-way ANOVA, $F_{(2,21)}=$ $\left.5.06, p<0.05, \eta^{2}=0.33\right)$ CS presentations compared with the control group (Fig. 1D), CaN levels increased only after the presentation of 10 CSs $\left(p<0.01\right.$, vs Ctr; one-way ANOVA, $F_{(2,21)}=4.77$, $p<0.05, \eta^{2}=0.31$; Fig. $\left.1 E\right)$.

Thus, while the increase in pERK $1 / 2$ in the BLA is a common correlate of both reconsolidation and extinction of fear memory, CaN levels are increased only when an extinction memory is being consolidated. This lack of specific association of the pERK1/2 signal with either reconsolidation or extinction indicates that this particular signaling pathway is not a molecular candidate subserving the transition between these memory processes. By contrast, the increase in CaN levels in the BLA emerges as a distinctive and specific characteristic of extinction memory consolidation, and therefore may be required for the shift from maintenance to loss of fear responses that follows increasing numbers of presentations of the fear CS. 
$10 \mathrm{CS}$ groups

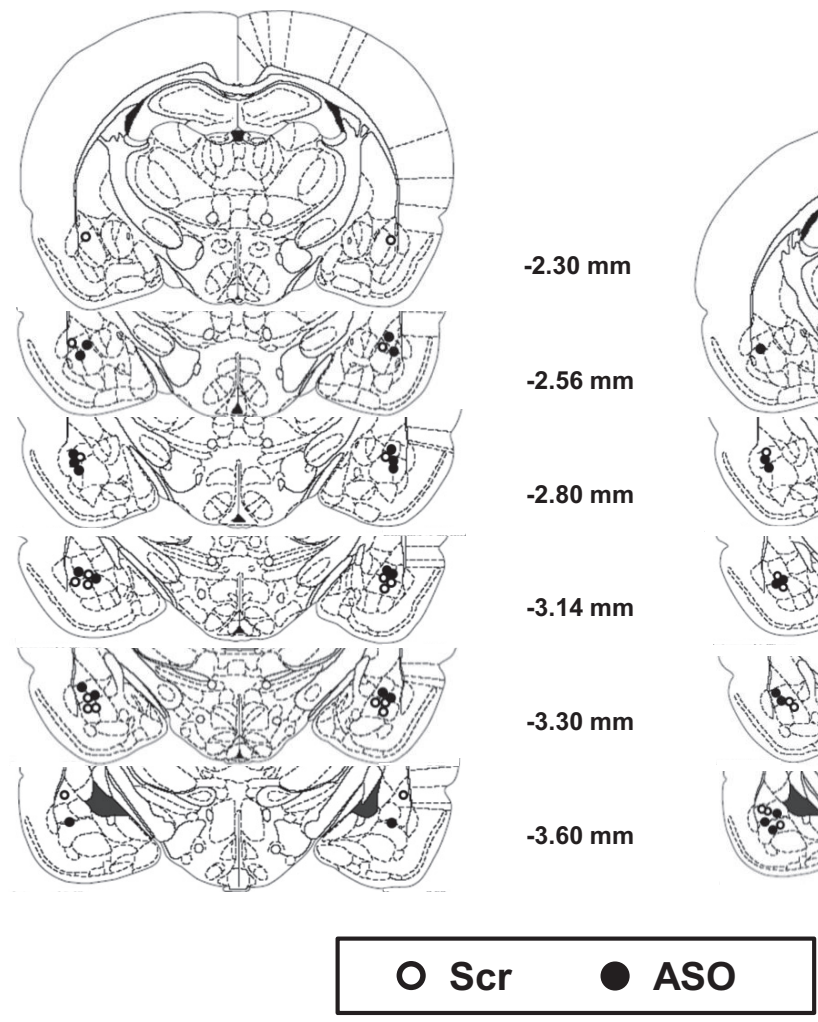

Figure 2. Schematic representation of the brain showing the placement of the infusion sites in animals in experiments described in Figure 3 (1CS and 10CS groups). All injections sites were within the BLA. Open circles, Scr ODN injections; closed circles, CaN ASO injections. Coordinates are given as distance from bregma. This figure was modified, with permission, from Paxinos and Watson (1998).

\section{High calcineurin levels in the BLA induced by repeated CS presentations are required for consolidation of fear extinction memory}

In the next set of experiments, we tested the hypothesis of a causal relationship between the increase in CaN levels in the BLA induced by 10 CS presentations and the establishment of a longlasting extinction memory. We hypothesized that the increase in synthesis of $\mathrm{CaN}$ in the BLA is required for the consolidation of the extinction memory, but is not required for reconsolidation of the original fear memory. An antisense oligodeoxynucleotide (ASO) was infused into the BLA to prevent the increase in CaN levels induced by the presentation of 10 CSs in animals with a fully consolidated fear memory. This enabled the evaluation of the role of $\mathrm{CaN}$ in the consolidation of a fear extinction memory. We also predicted that knockdown of CaN would have no impact on the reconsolidation process.

BLA-cannulated rats were trained with the presentation of two pairings of CS and footshock US, with all animals showing a higher level of freezing to the second CS compared with the first $\left(F_{(1,34)}=216.08, p<0.001, \eta^{2}=0.86\right.$; see Fig. $\left.3 A\right)$, and with no differences between groups $(F<1)$. The following day, the rats received an infusion into the BLA (Fig. 2) of either Scr ODNs or CaN ASOs $2.5 \mathrm{~h}$ before the presentation of 1 or $10 \mathrm{CSs}$. Even though the freezing levels were somewhat but equally reduced by the administration of either the Scr or CaN ASO into the BLA (Fig. 3A), all groups showed retention of fear memory, as indicated by the high level of freezing to the first $\operatorname{CS}\left(F_{(1,34)}=25.86\right.$, $\left.p<0.001, \eta^{2}=0.43\right)$, with no differences between groups $(F<$ 1). Within-session extinction by presentation of $10 \mathrm{CSs}$ was iden-
1 CS groups

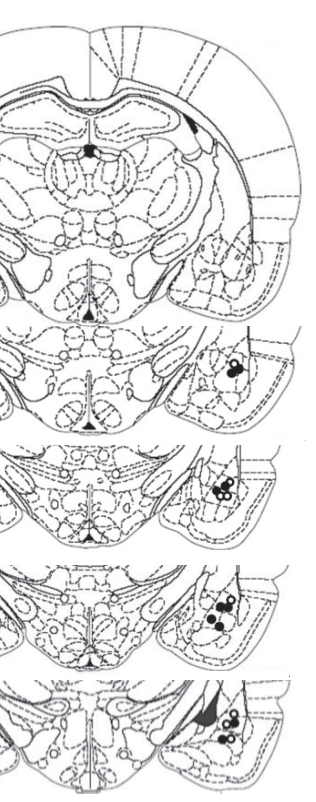

ASO and Scr groups that showed a significant decrease in freezing $\left(F_{(9,153)}=7.13, p<0.001, \eta^{2}=0.30\right.$; Fig. $3 A$, Re-exposure), with no differences between groups $(F<1)$. To allow for the complete clearance of the oligodeoxynucleotide from the BLA, the LTM-TS was conducted $4 \mathrm{~d}$ later. At LTM-TS, a two-way ANOVA revealed a significant effect of CS $\left(F_{(1,34)}=9.65, p<0.005, \eta^{2}\right.$ $=0.22)$ and no effect of drug $\left(F_{(1,34)}=\right.$ 3.31, $p=0.078)$, but, importantly, there was a significant interaction (CS $\times$ drug: $\left.F_{(1,34)}=9.65, p<0.005, \eta^{2}=0.22\right)$. Simple main-effects analysis revealed a significant differences between 10CS groups treated with Scr ODNs and ASO $\left(F_{(1,18)}=\right.$ 16.92, $p<0.005, \eta^{2}=0.48$; Fig. 3A, LTMTS) but no differences between the 1CS groups ( $F<1$; Fig. $3 A$, LTM-TS).

To confirm that the infusion of $\mathrm{CaN}$ ASO into the BLA prevented the increase in the level of phosphatase induced by the extinction protocol, animals were trained with 2 CS-US pairings (effect of CS-US: $F_{(1,7)}=25.86, p<0.001, \eta^{2}=0.79$; no effect of group: $F<1$ ) and 24 h later were divided into two groups that received an infusion into the BLA of either the ASO or Scr ODN solutions, followed $2.5 \mathrm{~h}$ later by the presentation of 10 CSs (Fig. 3B). As in the previous experiment, even though the freezing levels were partly reduced by the administration of either the Scr ODN or CaN ASO into the BLA, both groups nevertheless showed good retention of the fear memory $\left(F_{(1,7)}=15.89, p<0.01, \eta^{2}=\right.$ $0.69)$, with no differences between Scr ODN and ASO groups $\left(F_{(1,7)}=1.87, p=0.21\right)$ and a within-session decrease in freezing $\left(F_{(9,63)}=4.50, p<0.001, \eta^{2}=0.39\right)$ that again did not differ between groups $(F<1$; Fig. $3 B)$. One hour after the extinction protocol, the animals were killed, and cytoplasmic protein extracts from the BLA were prepared. Western blot analysis revealed a lower level of $\mathrm{CaN}$ in the ASO group compared with the Scr group ( $p<0.05$; Fig. $3 B$, bar graph), confirming that the microinfusion of the CaN ASO into the BLA prevented the synthesis de novo of the phosphatase induced by the extinction protocol.

The use of ODN to knock down the expression of a particular protein has the potential to produce nontarget effects. We therefore used an experimental design that also measured levels of an unrelated protein ( $\beta$-actin; Fig. $2 B$ ) following the infusion of $\mathrm{CaN}$ ASO in the BLA to assess its selectivity for $\mathrm{CaN}$ and reduce the likelihood of a nontarget effect. The results strongly support a specific effect of the ASO on CaN levels and hence on memory extinction.

Previous reports show that while BLA inactivation following the infusion of the $\mathrm{GABA}_{\mathrm{A}}$ receptor agonist muscimol during extinction training prevents fear expression and extinction learning (Laurent et al., 2008), the AMPA receptor antagonist LY293558 infused into the BLA prevents the expression of fear, but does not prevent labilization and restabilization of the fear memory (Milton et al., 2013). Although ODN microinfusion into the BLA before retrieval partially affected fear expression, the 
effect of CaN knockdown on memory extinction cannot be attributed to general BLA inactivation during CS presentations, since both the Scr and ASO groups showed markedly and significantly different levels of freezing at LTM-TS, with the Scr group showing complete fear extinction. Together, these data indicate that specifically blocking the increase in CaN levels in the BLA induced by the 10 CS extinction protocol completely prevented the consolidation of the fear extinction memory, as well as showing that the increase in CaN could not simply have reflected the mere presentation of an auditory stimulus having no association with the fearful US. Given the lack of any drug or CS-drug interaction between Scr ODN and ASO groups under the 10 CS protocol, it can be concluded that $\mathrm{CaN}$ knockdown into the BLA did not affect extinction acquisition. Furthermore, the reconsolidation process induced by one CS presentation was unaffected by $\mathrm{CaN}$ knockdown.

Gradual increases in the number of fear CS presentations result in a "dosedependent" transition from fear maintenance to fear inhibition

To further specify the behavioral and molecular characteristics of the transition from reconsolidation-inducing to extinctioninducing conditions, we analyzed the effect of increasing the number of CS presentations in animals with a fully consolidated fear memory on both conditioned freezing and levels of $\mathrm{CaN}$ in the BLA.

Two pairings of a clicker CS and shock resulted, as before, in a significant increase in freezing on subsequent presentations of the CS $\left(F_{(1,28)}=324.11, p<0.001, \eta^{2}=0.92\right)$, with no differences between groups $\left(F_{(3,28)}=1.84, p=0.163\right.$; Fig. $\left.4 A\right)$. On the following day, animals in each of the four groups were presented with $1,4,7$, or $10 \mathrm{CSs}$ (ITI $=1 \mathrm{~min})$. All groups showed fear memory retention, with a high level of freezing to the first CS $\left(F_{(1,28)}=477.07, p<0.001, \eta^{2}=0.95\right)$, with no differences between groups $(F<1)$. There was a decrease in freezing to the CS in the $4 \mathrm{CS}\left(F_{(3,21)}=7.43, p<0.001, \eta^{2}=0.51\right), 7 \mathrm{CS}\left(F_{(6,42)}\right.$ $\left.=10.72, p<0.001, \eta^{2}=0.61\right)$, and 10CS $\left(F_{(9,63)}=15.00, p<\right.$ $\left.0.001, \eta^{2}=0.68\right)$ groups (Fig. $4 A$, Re-exposure). Twenty-four hours later, freezing was measured following the presentation of 1 CS (LTM-TS). Freezing decreased proportionally in relation with the degree of CS exposure $\left(F_{(3,28)}=6.11, p<0.005, \eta^{2}=0.39\right)$. Thus, further post hoc analysis revealed that the level of freezing at the LTM-TS in the 1CS group was similar to that in the 4CS group $(p=0.32)$, but was significantly higher than in the 7 and 10 CS groups ( $p<0.05$ and $p<0.005$, respectively), while the level of freezing of the 10CS group was not different from that in the $4 \mathrm{CS}$ or 7CS groups ( $p=0.1$ and $p=0.52$, respectively; Fig. $4 A$ ). A positive correlation was observed between the level of freezing at the last CS of the re-exposure session and the level of freezing at LTM-TS $(r=0.710, p<0.01$; Fig. $4 B)$.
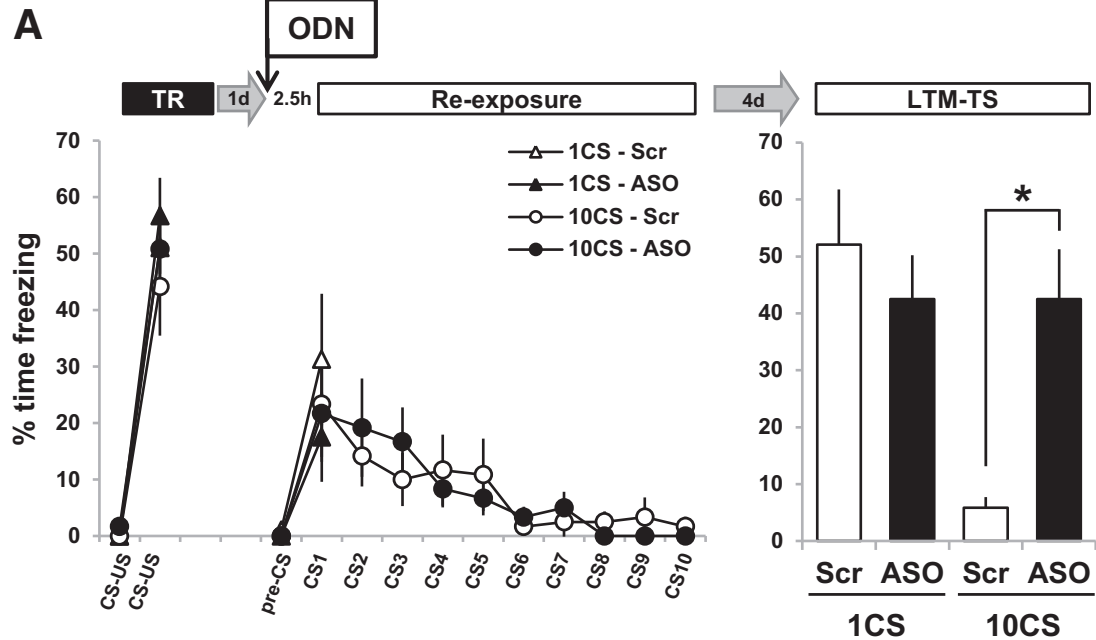

Figure 3. A CaN increase induced by $10 \mathrm{CS}$ s is required for consolidation of the fear extinction memory. $A$, Animals with fully of $1 \mathrm{CS}$ ( $n=10$ per group) or $10 \mathrm{CSs}$ (Scr ODN, $n=10 ; \mathrm{ASO}, n=8)$. Four days later, all groups were tested for long-term fear or extinction memory retention by the presentation of one $C S^{*}{ }^{*} p<0.005$. $\boldsymbol{B}$, Animals with fully consolidated fear memory were were killed and the BLA cytoplasmic extract was obtained. A representative result of the Western blot analysis along with the quantification of CaN levels in the BLA is shown. Data are presented as means \pm SEM. ${ }^{*} p<0.05$.

To analyze the effect of the number of CS presentations at retrieval on levels of $\mathrm{CaN}$ in the BLA, additional groups of fearconditioned animals were presented with $1,4,7$, or 10 CSs and were killed $1 \mathrm{~h}$ later (Fig. 4C). To control for the level of $\mathrm{CaN}$ in the BLA as a consequence of the consolidation of the original fear memory, the experiment included a control group that was also conditioned, but remained in the home cage until killed $24 \mathrm{~h}$ later. As expected, there was an effect of the number of CSs presented during training $\left(F_{(1,50)}=357.98, p<0.001, \eta^{2}=0.88\right)$, with no differences between groups $(F<1)$. The following day, all the CS-exposed groups showed fear memory retention $\left(F_{(1,35)}=\right.$ 444.26, $p<0.001, \eta^{2}=0.93$ ), with no differences between groups $(F<1$, Fig. $4 C, C S 1)$. ANOVA revealed an effect of group on CaN levels in the BLA $\left(F_{(4,50)}=7.23, p<0.001\right.$, $\eta^{2}=0.37$; Fig. $4 C$, bar graph). Post hoc analysis showed that CaN levels were higher and significantly different from controls only in the 10CS group $(p<0.001)$. A more detailed analysis at the individual level revealed a negative correlation between the level of freezing at the last CS of the exposure session and the level of CaN in the BLA $(r=-0.325, p<0.05$; Fig. 4D).

These data revealed a monotonic relationship between the number of CS presentations during the re-exposure session and 
A
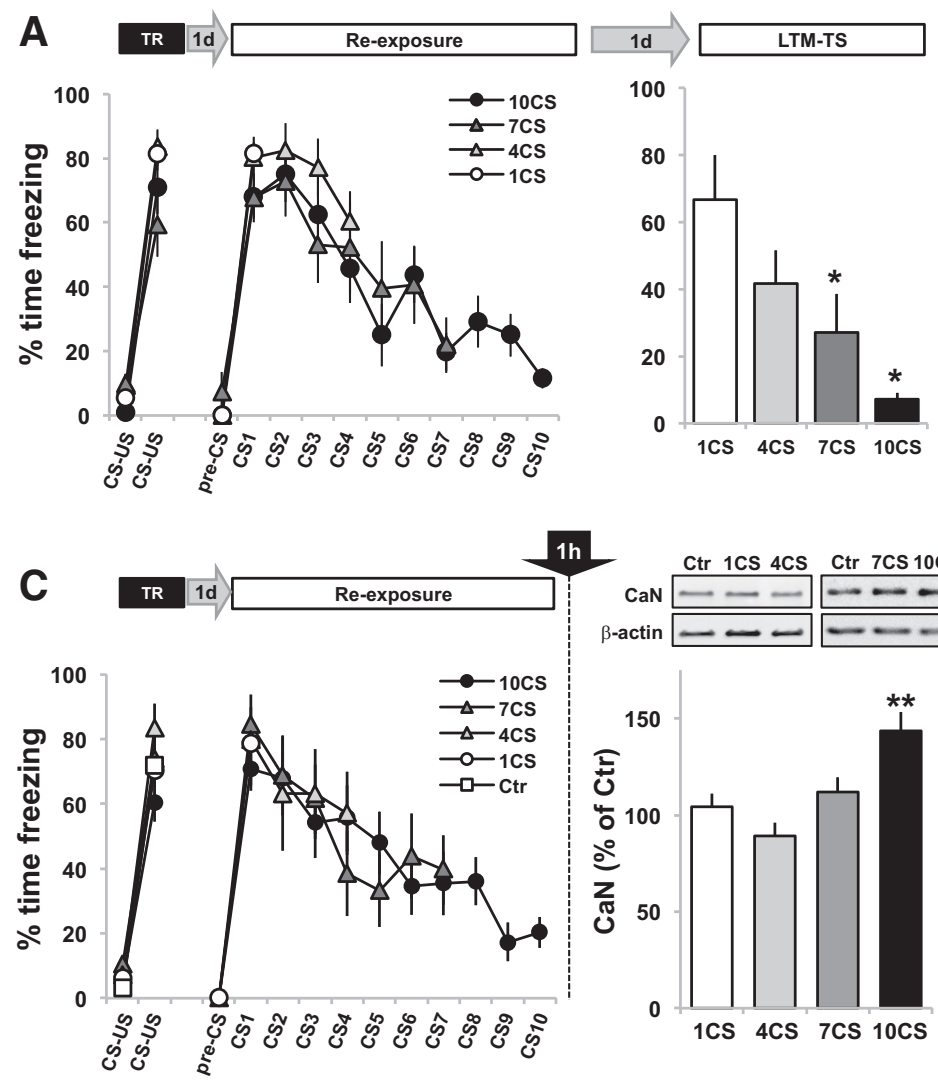

B
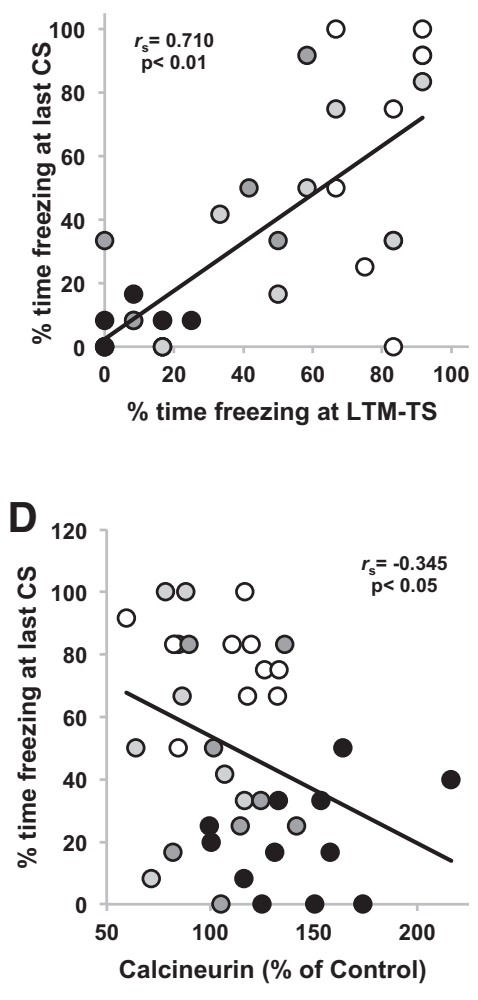

Figure 4. BLA CaN levels are negatively correlated with the level of conditioned freezing. $\boldsymbol{A}$, Animals with a fully consolidated fear memory were re-exposed to $1,4,7$, or $10 \mathrm{CSs}$ and tested $24 \mathrm{~h}$ later ( $n=8$ per group). ${ }^{*} p<0.05$ vs 1 CS group. $\boldsymbol{B}$, Level of freezing at the last CS of the re-exposure session versus the level of freezing at the LTM-TS. $\boldsymbol{C}$, Identical experimental procedure as in $\boldsymbol{A}$, but animals were killed $1 \mathrm{~h}$ after the presentation of $1 \mathrm{CS}(n=12), 4 \mathrm{CSs}(n=7), 7 \mathrm{CSs}(n=8)$, or 10 CSs $(n=12)$ along with a Ctr group ( $n=16)$ that received training the day before and was killed directly from the home cage. Left, Level of freezing during the training and CS presentation sessions. Right, Representative Western blot and level of cytosolic CaN in the BLA (Ctr group not shown). Data are presented as means \pm SEM. ${ }^{* *} p<0.01$. D , Correlation analysis between the level of CaN in the BLA and the percentage of time freezing at the last CS of the re-exposure session. Correlations were as follows: number of CS versus freezing level $\left(r_{(39)}=-0.734, p<0.01\right)$; number of CSs versus CaN level $\left(r_{(39)}=0.518, p<0.01\right)$; freezing level versus CaN level $\left(r_{(39)}=\right.$ $-0.345, p<0.05)$.

the level of freezing during testing, suggesting a gradual behavioral transition between reconsolidation-like and extinction-like mechanisms induced by repeated non-reinforced CS presentations, which is negatively correlated with a progressive increase in CaN levels in the BLA.

\section{NMDAR activity manipulation both modulates calcineurin levels in the BLA and alters the transition from reconsolidation to extinction}

In order to better understand the behavioral and molecular characteristics of the memory states that are transitional between reconsolidation and extinction, we took advantage of the bidirectional effects on these memory processes of NMDAR agonism and antagonism (Baker and Azorlosa, 1996; Walker et al., 2002; Suzuki et al., 2004; Lee et al., 2006). Using systemic administration of the NMDAR partial agonist DCS, and the antagonist MK-801, it was possible to reveal the dominant memory process in operation as well as the engagement of $\mathrm{CaN}$ synthesis induced by increasing the number of CS presentations at retrieval.

In accordance with our previous results (Lee et al., 2006), we expected to find bidirectional modulation of the level of freezing at the LTM-TS with the administration of the NMDAR partial agonist DCS or the antagonist MK-801. We predicted that DCS would enhance or maintain high levels of freezing if administered in conjunction with a 1 CS (reconsolidation) protocol, while MK-801 should have an amnestic effect, inducing a low level of freezing during the test. By contrast, if administered in conjunction with a 10 CS (extinction) protocol, DCS should result in low levels of freezing at LTM-TS by enhancing extinction, while MK801 should prevent extinction and result in a high level of freezing at test. However, the main objective of this experiment was to evaluate the impact of manipulating NMDAR activity at the molecular and behavioral levels on the transitional states between reconsolidation and extinction (i.e., the 4 and 7CS groups) in comparison with the expected effects of the treatments on 1 and 10CS groups.

Rats were fear conditioned as before with two CS-US pairings. The following day, the animals received an intraperitoneal injection of saline, DCS (15 mg/kg), or MK-801 (0.1 mg/kg), and 30 min later were exposed to 1,4 , 7, or 10 CS protocols (Fig. 5A). As predicted, the groups exposed to one CS showed a main effect of the drug at LTM-TS $\left(F_{(2,25)}=5.86, p<0.01, \eta^{2}=0.32\right)$, with the MK-801 group showing a lower level of freezing than the saline solution group ( $p<0.05$; Fig. $5 B$, bar graph), while in the $10 \mathrm{CS}$ groups there was a main effect of drug $\left(F_{(2,24)}=18.41, p<0.01\right.$, $\left.\eta^{2}=0.61\right)$, with the MK-801 group showing a higher level of freezing compared with the saline solution group $(p<0.01$; Fig. $5 E$, bar graph). The 7CS groups showed a main effect of drug $\left(F_{(2,22)}=18.23, p<0.01, \eta^{2}=0.62\right)$, with the MK-801 and DCS groups presenting a higher $(p<0.05)$ and lower $(p<0.01)$ level of freezing than the saline solution group, respectively (Fig. $5 D$, bar graph). The 4CS groups showed no differences in the level of 
A

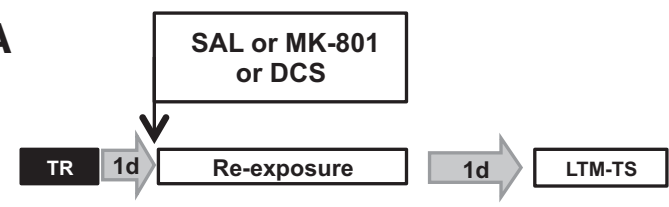

B
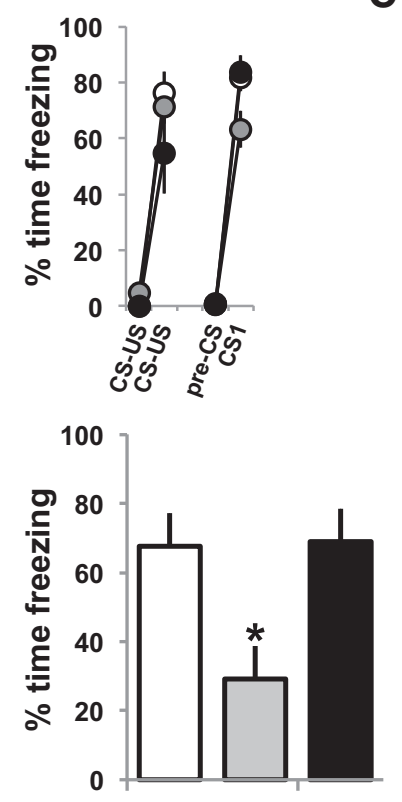

1CS
C
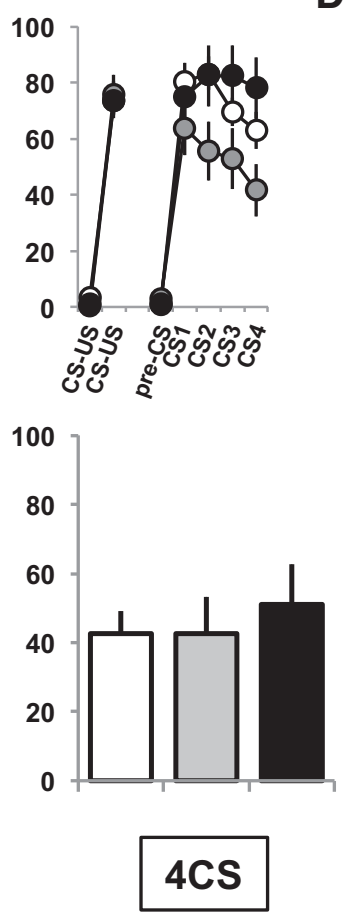

D
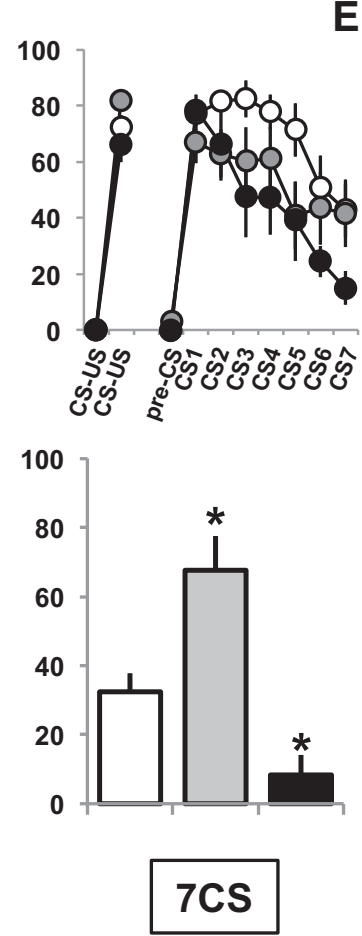

E
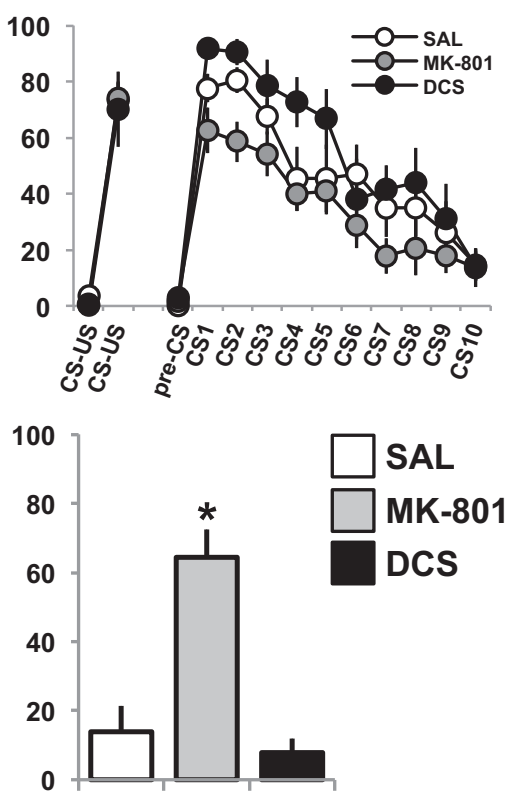

10CS

Figure 5. NMDAR agonist and antagonist treatments differentially affect the behavioral transition between reconsolidation and extinction. $A$, Experimental design. One day after training, the rats received an intraperitoneal injection of the NMDAR partial agonist DCS (15 mg/kg) or the antagonist MK-801 (0.1 mg/kg). $\boldsymbol{B}-\boldsymbol{E}, 0$ ne quarter of each group received 1 CS (B), 4 CS $(\boldsymbol{C}), 7(S)(\boldsymbol{D})$, or 10 CS $(\boldsymbol{E})$ presentations (line graphs). Twenty-four hours later, freezing behavior was measured in all groups by the presentation of one $C S($ bar graphs). Data are presented as means \pm SEM. MK-801 groups: $1 \mathrm{CS}, n=12 ; 4$ CSs, $n=9 ; 7 \mathrm{CSs}, n=9$; and 10 CSs, $n=11$. DCS groups: 1 CS, $n=7 ; 4$ CSs, $n=8 ; 7$ CS, $n=8 ;$ and 10 CSs, $n=8$. ${ }^{*} p<0.05$, ${ }^{* *} p<0.01$.

freezing at LTM-TS ( $F<1$; Fig. $5 C$, bar graph). Together, these data both confirm the utility of this approach in revealing the dominant memory process engaged by the different intermediate retrieval conditions, and also highlight the remarkable insensitivity of the 4CS-induced memory state to bidirectional manipulation of NMDAR activity.

To evaluate the effect of manipulating NMDAR activity on CaN levels in the BLA induced by increasing presentations of the fear CS (Fig. 4C), animals were trained as before, and the following day they received an intraperitoneal injection of either DCS or MK-801 30 min before the presentation of $1,4,7$ or 10 CSs, or were returned to the home cage (Ctr). One hour after the exposure session, the animals were killed, and extracts of BLA cytoplasmic proteins were prepared. Western blots revealed that treatment with $\mathrm{MK}-801$ prevented the increase in CaN levels in the BLA observed after increasing the number of CS presentations ( $F<1$; Fig. $6 A$, bar graph). Thus, systemic MK-801 administration prevented both memory extinction and $\mathrm{CaN}$ level increase in the BLA induced by increasing the number of CS presentations. On the contrary, Western blots revealed differences in CaN levels in DCS-treated groups $\left(F_{(4,49)}=3.61, p<\right.$ $0.05, \eta^{2}=0.24$; Fig. $6 B$, bar graph). Post hoc analysis revealed that in both the 7CS and 10CS groups there was a higher level of CaN compared with the control group ( $p<0.05$ in both), showing that the facilitatory effect of DCS on partial extinction induced by seven CSs is associated with a specific enhancement of the synthesis of $\mathrm{CaN}$ in the BLA.

Noninjected animals showed a significant increase in CaN levels in the BLA only after 10 CS presentations when compared with non-reactivated controls (Fig. 4). To analyze the effect of NMDAR activity manipulations on the modulation of CaN levels by increasing the number of CS presentations, we injected all groups with the NMDAR partial agonist DCS or the antagonist MK-801, and then compared the effect of CS presentations on CaN levels in the BLA with respect to their non-reactivated, DCS or MK-801 control group. This design thereby enabled us to quantify the interaction between memory retrieval and bidirectional NMDAR activity manipulations.

Therefore, NMDAR activity manipulation affected the 7CSinduced transitional state in a way similar in molecular and behavioral terms to the 10CS protocol, suggesting that $7 \mathrm{CS}$ presentations predominantly engage extinction-like mechanisms. In contrast, NMDAR manipulations did not affect the molecular and behavioral properties of the transitional state induced by the 4CS protocol, strongly suggesting that neither reconsolidation- nor extinction-like mechanisms are engaged. Together, these results show that increasing or decreasing NMDAR activity modulates both the behavioral transition of a fear memory from reconsolidation to extinction and also the engagement of CaN synthesis in the BLA. 
A
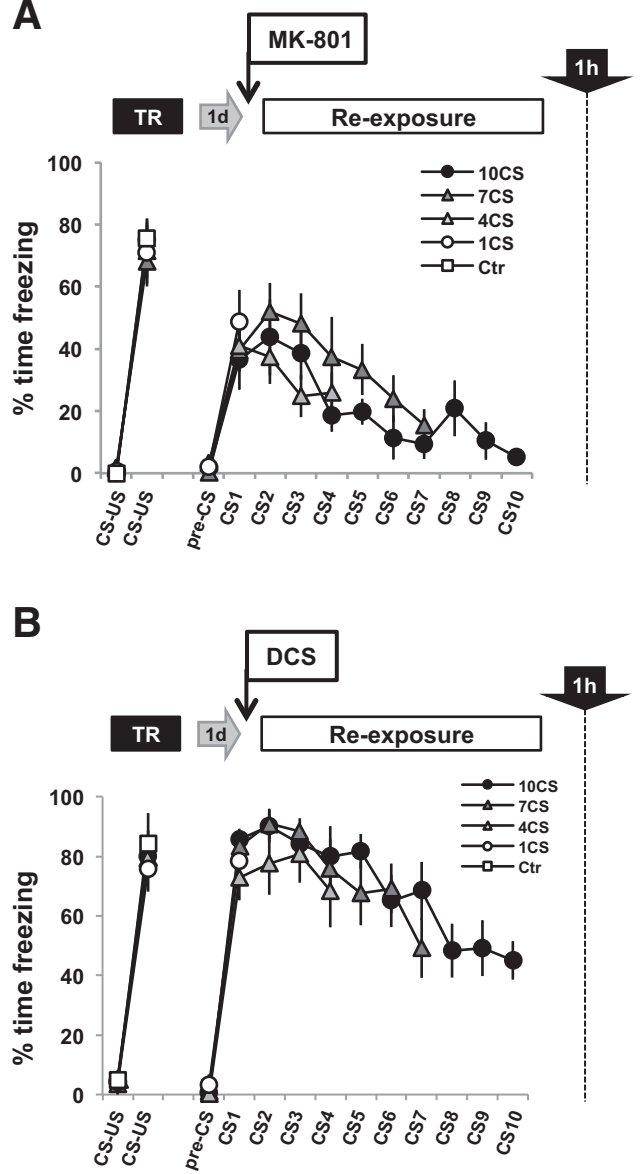
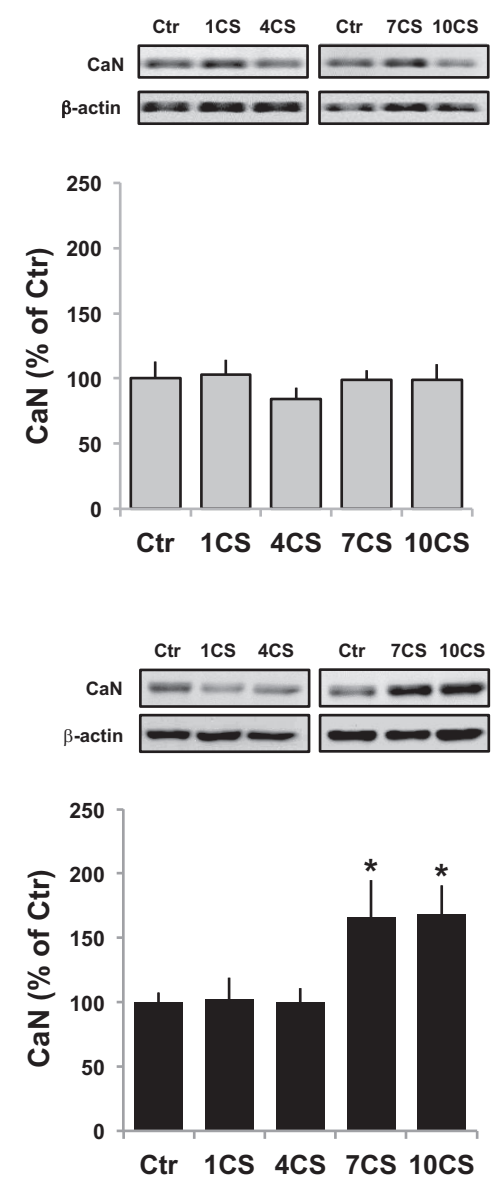

Figure 6. NMDAR agonist and antagonist treatments modulate levels of $\mathrm{CaN}$ in the BLA induced by increasing numbers of CS presentations. The procedure was as described in Figure 4 , but all animals were killed $1 \mathrm{~h}$ after the $\mathrm{CS}$ re-exposure session to quantify the levels of $\mathrm{CaN}$ in the BLA. $\boldsymbol{A}, \boldsymbol{B}$, As control groups, animals were trained, and $24 \mathrm{~h}$ later they were injected with MK-801 $(\boldsymbol{A})$ or DCS $(\boldsymbol{B})$ and were killed $90 \mathrm{~min}$ after from the home cage. Representative Western blot results are shown for each experiment. Bar graphs, Level of CaN in the BLA of animals receiving MK-801 $(\boldsymbol{A})$ or DCS $(\boldsymbol{B}) 30$ min before being presented with 1, 4, 7, or 10 CSs. MK-801 groups: Ctr group, $n=7 ; 1 C S$ group, $n=7 ; 4 C S$ group, $n=7 ; 7 C S$ group, $n=8$; and 10CS group, $n=8$. DCS groups: $n=10$ each. ${ }^{*} p<0.05$.

\section{Discussion}

This study demonstrates that reconsolidation and extinction of a fear CS memory are molecularly distinct and mutually exclusive processes. While the level of CaN in the BLA was increased by the non-reinforced presentation of 10 CSs (which resulted in fear extinction), but not by reactivation of fear by a single CS presentation (resulting in reconsolidation), pERK1/2 was increased in the BLA in both conditions. Preventing the increase in CaN levels in the BLA induced by 10 CS presentations by infusing a CaN antisense oligodeoxynucleotide specifically prevented the consolidation of the extinction memory, which depends, therefore, on the upregulation of cytosolic CaN. In contrast, $\mathrm{CaN}$ knockdown in the BLA during 1 CS presentation had no effect on fear memory reconsolidation. Moreover, repeated non-reinforced cue presentations induce a gradual behavioral transition between the maintenance and inhibition of fear that was negatively correlated with a progressive increase in $\mathrm{CaN}$ in the BLA. Modulation of NMDAR activity differentially affected the levels of $\mathrm{CaN}$ in the BLA and concomitantly affected the transition between reconsolidation and extinction induced by increasing the number of CS presentations. The NMDAR antagonist MK-801 prevented extinction and also the increase in CaN in the BLA induced by the strong extinction protocol (10 CSs), while the NMDAR partial agonist DCS enhanced fear extinction and induced a significant increase in $\mathrm{CaN}$ levels by the presentation of the partial extinction protocol (7 CS presentations). Thus, the increase in CaN synthesis in the BLA mediated by increased NMDAR activity is causally involved in the engagement of extinction mechanisms that result in the long-term loss of fear responding to the CS. At the behavioral level, NMDAR activity bidirectional manipulations showed that some of the transitional states between reconsolidationinducing and extinction-inducing retrieval conditions do not engage a dominant memory process. In the continuum of possible retrieval conditions, reconsolidation and extinction processes are mutually exclusive, separated by an insensitive phase where the amount of CS exposure terminates the labilization of the original memory, but is insufficient to trigger the formation of the extinction memory.

\section{Role of calcineurin in the persistence of the extinction memory}

Fully consolidated cued fear memories, if not retrieved, can persist for up to the entire life of an animal (Gale et al., 2004). If retrieved, reconsolidation or extinction of the original memory may occur depending on the degree of CS exposure (Eisenberg et al., 2003; Pedreira and Maldonado, 2003). There are partially overlapping molecular mechanisms underlying fear memory consolidation, reconsolidation, and extinction. For example, each process depends upon de novo protein synthesis and NMDAR activation (Davis and Squire, 1984; Miserendino et al., 1990; Nader et al., 2000; Vianna et al., 2001; Lee et al., 2006). However, reconsolidation and consolidation are also dissociable at the molecular level (Lee et al., 2004). Here we have shown that ERK1/2 activation in the BLA occurs during both reconsolidation and extinction, consistent with data showing that ERK1/2 activity is required for both processes (Duvarci et al., 2005; Herry et al., 2006). By contrast, CaN affects the consolidation of both the original and the extinction memory in completely opposite ways. Inhibiting or reducing the level of $\mathrm{CaN}$ during training of the original memory facilitates fear memory consolidation (Ikegami and Inokuchi, 2000; Baumgärtel et al., 2008), suggesting that $\mathrm{CaN}$ can modulate the establishment of aversive memories. Here we show for the first time that knocking down CaN during reconsolidation had no effect on the original memory, suggesting that the updating process induced by memory reactivation does not require $\mathrm{CaN}$, which is instead involved in establishing the fear memory (Ikegami and Inokuchi, 2000). In contrast, CaN knockdown in the BLA during extinction training specifically inhibited the consolidation of extinction of the aversive memory without affecting either the expression of fear or within-session extinction (Fig. $3 A$ ). It is of note that intra-BLA infusion of calcineurin activity inhibitors FK-506 and cypermethrin before extinction have 
been shown to prevent the acquisition of an extinction memory (Lin et al., 2003).

Thus, the extinction protocol has a dual effect on $\mathrm{CaN}$ in the BLA: an increase in activity is required either for acquisition of the extinction memory or short-term extinction (Lin et al., 2003), but increased synthesis of new $\mathrm{CaN}$ is specifically required for the establishment of a persistent extinction memory (Figs. 1, 3). In vitro studies have further shown that the inhibition of $\mathrm{CaN}$ facilitates LTP (Ikegami et al., 1996) but inhibits the establishment of LTD (Mulkey et al., 1994). Together, these findings reveal distinctive molecular mechanisms underlying the formation and maintenance of excitatory and inhibitory memory traces. In the BLA, ERK1/2 activation may account for the maintenance of the excitatory component at reconsolidation, but also the formation of the new inhibitory trace during extinction, while CaN levels may govern the transition from reconsolidation to extinction, possibly by reducing the activation of the original memory trace in response to the CS.

Discrete subpopulations of amygdala neurons have been shown to encode either fear conditioning or extinction memories (Herry et al., 2008). After training, a CS presentation induces the activation of fear neurons, while repeated CS presentations result in the activation of extinction neurons and the inhibition of fear neurons (Herry et al., 2008). Our findings show that while reconsolidation induced by one CS is associated with potentiation-like mechanisms (e.g., ERK1/2 activation), extinction involves both potentiation- and depotentiation-associated mechanisms within the BLA. These data therefore emphasize the central role of the BLA in the maintenance or inhibition of fear, and the unique molecular landscapes associated with reconsolidation and extinction processes.

\section{Molecular and behavioral aspects of the transition from reconsolidation to extinction}

In the present study, we have revealed novel behavioral and molecular features of the transition between reconsolidation and extinction. Operationally, the use of stepwise increments in the number of discrete CS presentation events at retrieval provided a method with which to explore the transitional states between reconsolidation-induction and extinction-induction conditions, allowing precise control over the number of times that the prediction of the US by the CS failed. Gradually increasing the number of CS presentations "dose dependently" resulted in a decrease in the freezing response measured at testing. BLA CaN level was negatively correlated with the percentage of time spent freezing during the last CS presentation, with CaN levels being significantly increased only in the 10CS group. Enhancing NMDAR activity by systemic injection of DCS in animals receiving seven CS presentations resulted in a behavioral (complete extinction) and molecular (high BLA CaN level) pattern similar to that seen in the $10 \mathrm{CS}$ group, indicating that the dominant memory process induced by the seven CS presentations is extinction. In marked contrast to the $1 \mathrm{CS}$ group, the 4CS group was, however, completely insensitive to manipulations of the NMDAR, with MK-801- and DCS-injected groups showing a very similar level of freezing to that in the saline control group. This striking finding strongly suggests that the transitional state induced by four CSs engages neither reconsolidation nor extinction. Remarkably, while MK-801 completely blocked the increase in CaN by 10 CSs in the BLA and prevented the consolidation of extinction memory, and DCS induced an increase in CaN and enhanced extinction in the 7CS group, neither drug had any behavioral or molecular effects in the 4CS group. These data provide key evidence in support of the behavioral and molecular dissociation

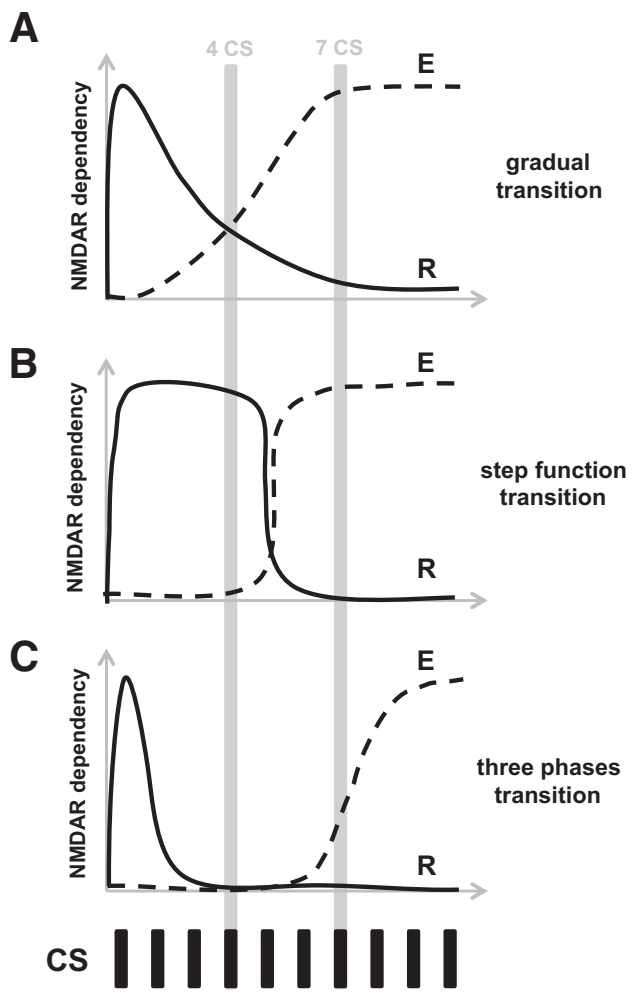

Figure 7. Models of the transition from reconsolidation to extinction of fear memories. $\boldsymbol{A}$ Gradual model. $\boldsymbol{B}$, Step function model. $\boldsymbol{C}$, Three-phase model. R, Reconsolidation process; $E$, extinction process; $y$-axis, sensitivity to NMDAR activity manipulations; $x$-axis, number of $C S$ presentations.

between the transitional states induced by exposure to four and seven CS presentations.

At least three alternative models can be used to describe the NMDAR-dependent transition from reconsolidation to extinction, as follows: (1) a gradual transition model, with the transitional states reflecting different degrees of engagement of one or the other process (Fig. 7A); (2) a step function transition model, with reconsolidation and extinction being mutually exclusive (Fig. 7B); and (3) a three-phase transition model (Fig. 7C). While the first two models fail to predict the lack of significant behavioral or molecular effect of bidirectional manipulation of NMDAR activity in the 4CS group, the experimental results favor the three-phase transition model. Hence, a single or few CS presentations (fewer than four) will induce the labilization and subsequent reconsolidation of the original fear memory; further presentations of the CS (e.g., four presentations) cancels the lability of the original memory and prevents reconsolidation, but fails to engage extinction. Finally, a greater number of CS presentations (7-10 CS presentations) gradually engages extinction. Interestingly, it has been demonstrated that a second CS presentation can nullify the protein synthesis inhibition sensitivity of a fear memory induced $1 \mathrm{~h}$ earlier by the first CS presentation (Jarome et al., 2012). The proposed molecular mechanisms responsible for apparently cancelling or nullifying the labilization processes that are initiated by early presentation of the CS are totally unknown. The presentation of a second, third, or fourth CS (in our procedure) could trigger an interfering mechanism that might terminate the ubiquitin-proteasome system (UPS)dependent labilization of the original memory induced by the early CS presentation (Lee et al., 2008), perhaps by local activation of deubiquitinating enzymes (Hegde, 2010) that previously 
have been associated with UPS homeostasis regulation in synaptic terminals (Cartier et al., 2009). Together, these data strongly suggest that reconsolidation and extinction of a CS fear memory are mutually exclusive and that some transitional states induced by increasing the numbers of non-reinforced CS presentations engage neither mechanism, reflecting a transitional or "limbo" state between updating, or reconsolidation, and inhibition through extinction learning.

Our present results demonstrate that the increase in CaN levels in the basolateral amygdala is uniquely and causally related to extinction. We have shown that reconsolidation and extinction of the cued fear memory are mutually exclusive, having distinct molecular characteristics, and we have also revealed that there is a novel insensitive, or limbo, state of the original memory that separates these processes.

\section{References}

Altschul SF, Madden TL, Schäffer AA, Zhang J, Zhang Z, Miller W, Lipman DJ (1997) Gapped BLAST and PSI-BLAST: a new generation of protein database search programs. Nucleic Acids Res 25:3389-3402. CrossRef Medline

Baker JD, Azorlosa JL (1996) The NMDA antagonist MK-801 blocks the extinction of Pavlovian fear conditioning. Behav Neurosci 110:618-620. CrossRef Medline

Baumgärtel K, Genoux D, Welzl H, Tweedie-Cullen RY, Koshibu K, Livingstone-Zatchej M, Mamie C, Mansuy IM (2008) Control of the establishment of aversive memory by calcineurin and Zif268. Nat Neurosci 11:572-578. CrossRef Medline

Blanchard RJ, Blanchard DC (1969) Crouching as an index of fear. J Comp Physiol Psychol 67:370-375. CrossRef Medline

Bouton ME (1991) Context and retrieval in extinction and in other examples of interference in simple associative learning. In: Current topics in animal learning: brain, emotion and cognition (Dachowski L, Flaherty CF, eds), pp 25-53. Hillsdale, NJ: Erlbaum.

Campeau S, Davis M (1995) Involvement of the central nucleus and basolateral complex of the amygdala in fear conditioning measured with fearpotentiated startle in rats trained concurrently with auditory and visual conditioned stimuli. J Neurosci 15:2301-2311. Medline

Cartier AE, Djakovic SN, Salehi A, Wilson SM, Masliah E, Patrick GN (2009) Regulation of synaptic structure by ubiquitin C-terminal hydrolase L1. J Neurosci 29:7857-7868. CrossRef Medline

Davis HP, Squire LR (1984) Protein synthesis and memory: a review. Psychol Bull 96:518-559. CrossRef Medline

de la Fuente V, Freudenthal R, Romano A (2011) Reconsolidation or extinction: transcription factor switch in the determination of memory course after retrieval. J Neurosci 31:5562-5573. CrossRef Medline

Dunn LT, Everitt BJ (1988) Double dissociations of the effects of amygdala and insular cortex lesions on conditioned taste aversion, passive avoidance, and neophobia in the rat using the excitotoxin ibotenic acid. Behav Neurosci 102:3-23. CrossRef Medline

Duvarci S, Nader K, LeDoux JE (2005) Activation of extracellular signalregulated kinase- mitogen-activated protein kinase cascade in the amygdala is required for memory reconsolidation of auditory fear conditioning. Eur J Neurosci 21:283-289. CrossRef Medline

Eisenberg M, Kobilo T, Berman DE, Dudai Y (2003) Stability of retrieved memory: inverse correlation with trace dominance. Science 301:11021104. CrossRef Medline

Gale GD, Anagnostaras SG, Godsil BP, Mitchell S, Nozawa T, Sage JR, Wiltgen B, Fanselow MS (2004) Role of the basolateral amygdala in the storage of fear memories across the adult lifetime of rats. J Neurosci 24:3810-3815. CrossRef Medline

Garver TD, Kincaid RL, Conn RA, Billingsley ML (1999) Reduction of calcineurin activity in brain by antisense oligonucleotides leads to persistent phosphorylation of tau protein at Thr181 and Thr231. Mol Pharmacol 55:632-641. Medline

Hegde AN (2010) The ubiquitin-proteasome pathway and synaptic plasticity. Learn Mem 17:314-327. CrossRef Medline

Herry C, Trifilieff P, Micheau J, Lüthi A, Mons N (2006) Extinction of auditory fear conditioning requires MAPK/ERK activation in the basolateral amygdala. Eur J Neurosci 24:261-269. CrossRef Medline
Herry C, Ciocchi S, Senn V, Demmou L, Müller C, Lüthi A (2008) Switching on and off fear by distinct neuronal circuits. Nature 454:600-606. CrossRef Medline

Ikegami S, Inokuchi K (2000) Antisense DNA against calcineurin facilitates memory in contextual fear conditioning by lowering the threshold for hippocampal long-term potentiation induction. Neuroscience 98:637646. CrossRef Medline

Ikegami S, Kato A, Kudo Y, Kuno T, Ozawa F, Inokuchi K (1996) A facilitatory effect on the induction of long-term potentiation in vivo by chronic administration of antisense oligodeoxynucleotides against catalytic subunits of calcineurin. Brain Res Mol Brain Res 41:183-191. CrossRef Medline

Jarome TJ, Kwapis JL, Werner CT, Parsons RG, Gafford GM, Helmstetter FJ (2012) The timing of multiple retrieval events can alter GluR1 phosphorylation and the requirement for protein synthesis in fear memory reconsolidation. Learn Mem 19:300-306. CrossRef Medline

Laurent V, Marchand AR, Westbrook RF (2008) The basolateral amygdala is necessary for learning but not relearning extinction of context conditioned fear. Learn Mem 15:304-314. CrossRef Medline

LeDoux JE (2000) Emotion circuits in the brain. Annu Rev Neurosci 23: 155-184. CrossRef Medline

Lee JL, Everitt BJ, Thomas KL (2004) Independent cellular processes for hippocampal memory consolidation and reconsolidation. Science 304: 839-843. CrossRef Medline

Lee JL, Milton AL, Everitt BJ (2006) Reconsolidation and extinction of conditioned fear: inhibition and potentiation. J Neurosci 26:10051-10056. CrossRef Medline

Lee SH, Choi JH, Lee N, Lee HR, Kim JI, Yu NK, Choi SL, Lee SH, Kim H, Kaang BK (2008) Synaptic protein degradation underlies destabilization of retrieved fear memory. Science 319:1253-1256. CrossRef Medline

Lin CH, Yeh SH, Leu TH, Chang WC, Wang ST, Gean PW (2003) Identification of calcineurin as a key signal in the extinction of fear memory. J Neurosci 23:1574-1579. Medline

Malleret G, Haditsch U, Genoux D, Jones MW, Bliss TV, Vanhoose AM, Weitlauf C, Kandel ER, Winder DG, Mansuy IM (2001) Inducible and reversible enhancement of learning, memory, and long-term potentiation by genetic inhibition of calcineurin. Cell 104:675-686. CrossRef Medline

Mao SC, Hsiao YH, Gean PW (2006) Extinction training in conjunction with a partial agonist of the glycine site on the NMDA receptor erases memory trace. J Neurosci 26:8892-8899. CrossRef Medline

Milton AL, Merlo E, Ratano P, Gregory BL, Dumbreck JK, Everitt BJ (2013) Double dissociation of the requirement for GluN2B- and GluN2Acontaining NMDA receptors in the destabilization and restabilization of a reconsolidating memory. J Neurosci 33:1109-1115. CrossRef Medline

Miserendino MJ, Sananes CB, Melia KR, Davis M (1990) Blocking of acquisition but not expression of conditioned fear-potentiated startle by NMDA antagonists in the amygdala. Nature 345:716-718. CrossRef Medline

Mulkey RM, Endo S, Shenolikar S, Malenka RC (1994) Involvement of a calcineurin/inhibitor-1 phosphatase cascade in hippocampal long-term depression. Nature 369:486-488. CrossRef Medline

Nader K, Schafe GE, LeDoux JE (2000) Fear memories require protein synthesis in the amygdala for reconsolidation after retrieval. Nature 405:722726. CrossRef Medline

Pavlov IP (1927) Conditioned reflexes: an investigation of the physiological activity of the cerebral cortex. London: Oxford UP.

Paxinos G, Watson C (1998) The rat brain in stereotaxic coordinates, Ed 4. New York: Academic.

Pedreira ME, Maldonado H (2003) Protein synthesis subserves reconsolidation or extinction depending on reminder duration. Neuron 38:863-869. CrossRef Medline

Suzuki A, Josselyn SA, Frankland PW, Masushige S, Silva AJ, Kida S (2004) Memory reconsolidation and extinction have distinct temporal and biochemical signatures. J Neurosci 24:4787-4795. CrossRef Medline

Sweatt JD (2004) Mitogen-activated protein kinases in synaptic plasticity and memory. Curr Opin Neurobiol 14:311-317. CrossRef Medline

Vianna MR, Szapiro G, McGaugh JL, Medina JH, Izquierdo I (2001) Retrieval of memory for fear-motivated training initiates extinction requiring protein synthesis in the rat hippocampus. Proc Natl Acad Sci U S A 98:12251-12254. CrossRef Medline

Walker DL, Ressler KJ, Lu KT, Davis M (2002) Facilitation of conditioned fear extinction by systemic administration or intra-amygdala infusions of D-cycloserine as assessed with fear-potentiated startle in rats. J Neurosci 22:2343-2351. Medline 